\title{
Acute Subdural Hematoma Immediately After Extended Transsphenoidal Surgery for Craniopharyngioma
}

\author{
Noriaki FUKUHARA, Hiroshi NISHIOKA, Shozo YAMADA \\ Toranomon Hospital, Department of Hypothalamic and Pituitary Surgery, Tokyo, Japan
}

\section{ABSTRACT}

We report the first case of postoperative acute subdural hemorrhage following extended transsphenoidal surgery (TSS). The patient was a 74-year-old woman with a cystic suprasellar craniopharyngioma with panhypopituitarism. Extended TSS resulted in complete tumor removal. However her consciousness did not recover well. Emergent computed tomography demonstrated a right acute supratentorial subdural hematoma with impending cerebral herniation. Emergent craniotomy revealed hemorrhage from the vein of Labbé. It may have been caused by acute intracranial hypotension secondary to rapid and massive drainage of cerebrospinal fluid. Surgeons should be aware of the possibility of intracranial hemorrhage as a rare but critical complication of extended TSS.

KEYWORDS: Craniopharyngioma, Extended transsphenoidal surgery, Acute subdural hematoma, Complication

\section{INTRODUCTION}

$\mathrm{T}$ Transsphenoidal surgery (TSS) is the principal and standard operative technique for pituitary adenomas and other lesions in and around the sella turcica. It is a reasonably safe procedure with low morbidity, particularly in experienced hands. However, most craniopharyngiomas develop from the pituitary stalk as a suprasellar lesion and it is difficult to remove such masses by the conventional TSS approach. With the refinement of surgical instruments and techniques, extended TSS was introduced to treat suprasellar tumors (8). This approach directly exposes the suprasellar and retrochiasmatic regions without brain retraction and thus provides excellent exposure and accessibility for excision of most suprasellar craniopharyngiomas.

An acute subdural hematoma is a possible complication following craniotomy, but only four cases of subdural hematoma after TSS have been reported $(4,10,13,14)$. In all the four cases of subdural hematoma, the complication developed several days after surgery. We report the first case of acute subdural hematoma following extended transsphenoidal approach for surgical removal of a craniopharyngioma.

\section{CASE REPORT}

A 74-year-old woman had been suffering from appetite loss and depression for a year prior to examination and was diagnosed with craniopharyngioma, panhypopituitarism, and diabetes insipidus. Following hormonal replacement therapy, her appetite loss and depression improved. When the craniopharyngioma was first diagnosed, she did not have any visual disturbances. However, her vision deteriorated and she was referred to our hospital for surgical treatment. Her tumor was $30 \times 16 \times 15 \mathrm{~mm}$ in size, cystic, and extended from the sella to the third ventricle (Figure 1). She underwent extended TSS and the tumor was totally resected. The tumor mildly adhered to the floor of third ventricle, and the third ventricle was not opened. The operation was uneventfully completed, and any abnormal findings, for example cerebral edema or brain shift, were not revealed in the suprasellar region by endoscopy. Repair of the sellar dura mater was performed using a fascial patch graft. Despite this uneventful surgery, her recovery from general anesthesia was abnormally slow and she remained drowsy after extubation. About 30 minute after operation emergent computed tomography (CT) was performed and 

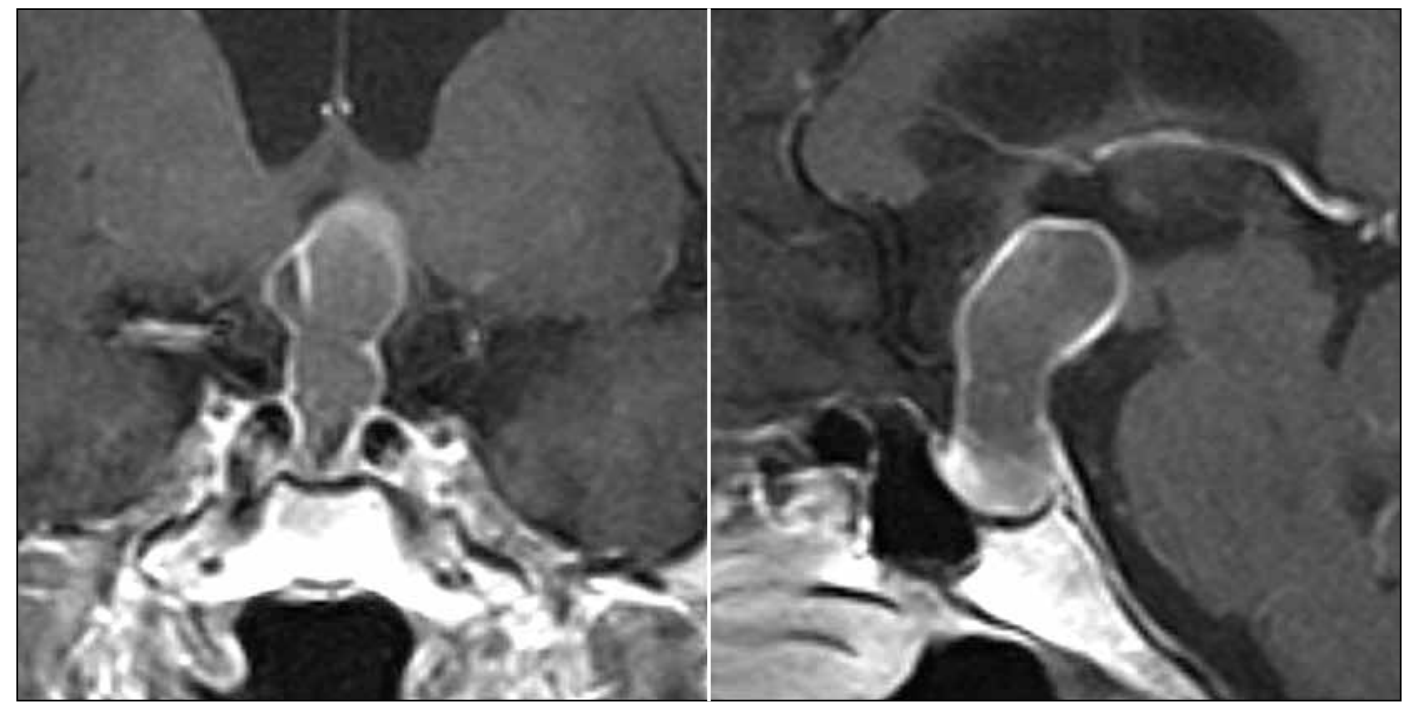

Figure 1: Preoperative MRI findings.

Left: coronal section.

Right: sagittal section.

A cystic

craniopharyngioma

extending from the sella

to the suprasellar region is observed.

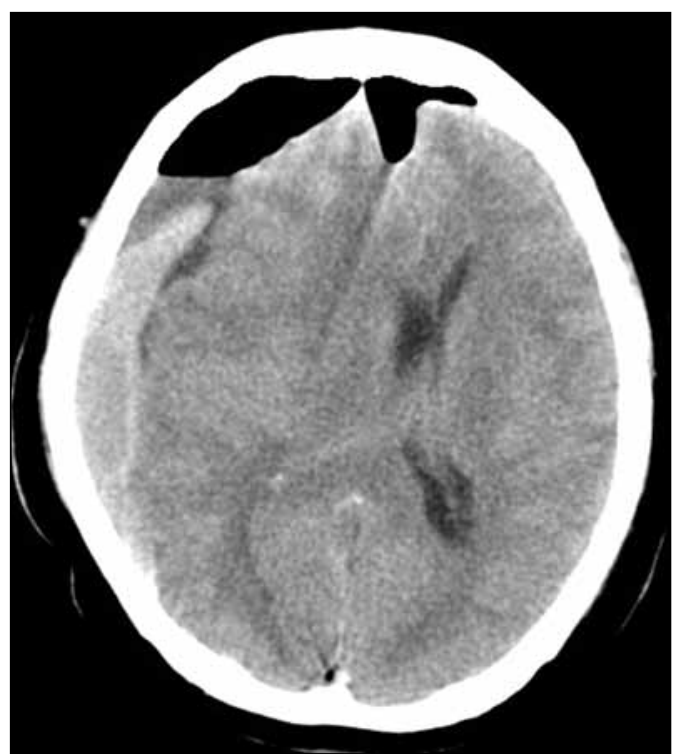

Figure 2: CT scan immediately after extended transsphenoidal surgery. A right subdural hematoma appears to compress the brain.

it revealed a right acute subdural hematoma (Figure 2). She immediately underwent emergency right craniotomy and evacuation of the hematoma. Bleeding from the vein of Labbé was confirmed during the surgery. Disturbances of consciousness continued for several weeks after surgery, and she did not regain the ability to walk unassisted for several months.

\section{DISCUSSION}

In this report, we reported the first case of postoperative acute subdural hemorrhage immediately after extended transsphenoidal surgery. Only four cases of the postoperative subdural hematoma after TSS have been reported in literatures, two of which were subacute or chronic subdural hematomas $(4,10,13,14)$. Tanaka et al. reported the case of a 74-year-old man with a postoperative chronic subdural hematoma diagnosed 78 days after extended TSS and the authors suggested that it was caused by postoperative subdural fluid collection (14). Sudhakar et al. reported the case of a 77-year-old man who underwent TSS for a nonfunctioning pituitary adenoma. A lumbar drainage tube was inserted on the second postoperative day because of cerebrospinal fluid (CSF) leakage. Two days after insertion of the tube, a subdural hematoma developed, likely due to intracranial hypotension. Evacuation of the enlarging subdural hematoma was performed by burr-hole surgery on the $15^{\text {th }}$ postoperative day (13). However chronic subdural hematoma following extended TSS may not be as rare as suggested by these sporadic reports. Both Frank et al. and de Devitiis et al. reported one chronic subdural hematoma in 10 cases craniopharyngioma following endoscopic extended TSS $(3,5)$. The two previous cases of acute subdural hematoma were attributed to postoperative CSF leak that developed several days after TSS rather than as a direct result of TSS. Both patients experienced rapid deterioration of consciousness several hours after the CSF leak and this was attributed to the rapid runoff of $\operatorname{CSF}(4,10)$. Subdural hematoma in these previous reports were subacute or chronic, or not related directly to operation. Acute subdural hematoma occurring immediately after transsphenoidal surgery has not been reported. Therefore, our case is the first report of acute subdural hematoma occurring intraoperatively in transsphenoidal surgery.

Acute intracranial subdural hematoma due to severe CSF drainage has also been reported during or immediately following other procedures; for example after lumbar drainage or during lumbar spinal surgery $(7,11,12)$. In these cases, CSF drainage resulted in severe intracranial hypotension that induced intracranial hemorrhage. In addition, intraoperative intracranial hemorrhages that were not associated with the operative site have been reported $(6,9)$. Tumor resection and hematoma evacuation relieve intracranial hypertension, but the radical decrease in intracranial pressure can induce intracranial 
hemorrhage. We believe that intracranial hypotension caused by rapid loss of CSF and tumor removal during extended TSS also lead to acute subdural hematoma in our case.

In the past, most craniopharyngiomas were removed by craniotomy, but the optic chiasm and other vital structures prevent surgeons from removing suprasellar craniopharyngiomas. Extended TSS was developed for such cases and has proven to be an effective and safe approach for suprasellar lesions because it allows a clear view of the lesion below the optic chiasm $(8,14)$. However, this approach is always complicated by intraoperative CSF leakage due to the dural incision of the suprasellar region, which must be repaired intraoperatively. Several procedures to repair the sella turcica were reported to prevent postoperative CSF leakage $(1,2,8,15)$, but it is impossible to avoid the intraoperative CSF leakage using TSS for suprasellar lesions. Thus, surgeons must avoid too high head positioning which causes excessive intracranial hypotension, and a quick dural incision which causes massive CSF flow out. For interval between CT scan from the end of surgery was short, the bleeding would have occurred during surgery, in this case. However, any abnormal finding was not detected by intraoperative microscopic or endoscopic view of operative field. It may be difficult to find out the development of subdural hematoma. CT scan should be performed immediately after extended TSS. Although this approach is recommended for removal of suprasellar craniopharyngiomas, surgeons must be aware of possible vascular complications such as acute subdural hematoma and patients should be informed of this critical and unpredictable complication.

Moreover, extended TSS was introduced as a less invasive approach for safe and effective resection of craniopharyngioma. However, in the present 75 year-old woman with atrophic cisterns and a completely cystic tumor, a pterional approach could have been relatively straightforward in achieving the goal and preventing this complication. The surgical approach should be selected according to each individual case.

\section{CONCLUSION}

Extended TSS will be popular for the treatment of craniopharyngiomas. Hence vascular complications, although extremely rare, should be recognized as a possible cause of poor prognosis.

\section{REFERENCES}

1. Ahn JY, Kim SH: A new technique for dural suturing with fascia graft for cerebrospinal fluid leakage in transsphenoidal surgery. Neurosurgery 65: 65-71, 2009
2. Cavallo LM, Messina A, Esposito F, de Divitiis O, Dal Fabbro M, de Divitiis E, Cappabianca P: Skull base reconstruction in the extended endoscopic transsphenoidal approach for suprasellar lesions. J Neurosurg 107: 713-720, 2007

3. de Divitiis E, Cappabianca P, Cavallo LM, Esposito F, de Divitiis O, Messina A: Extended endoscopic transsphenoidal approach for extrasellar craniopharyngiomas. Neurosurgery 61: 219-227, 2007

4. Eloqayli H, Cappelen J, Vik A: Acute spontaneous subdural haematoma after transsphenoidal surgery. Acta Neurochir (Wien) 148: 587-590, 2006

5. Frank G, Pasquini E, Doglietto F, Mazzatenta D, Sciarretta V, Farneti G, Calbucci F: The endoscopic extended transsphenoidal approach for craniopharyngiomas. Neurosurgery 59: 75-83, 2006

6. Fridley J, Thomas J, Kitagawa R, Chern J, Omeis I: Immediate development of a contralateral acute subdural hematoma following acute subdural hematoma evacuation. J Clin Neurosci 18: 422-423, 2011

7. Kim HJ, Cho YJ, Cho JY, Lee DH, Hong KS: Acute subdural hematoma following spinal cerebrospinal fluid drainage in a patient with freezing of gait. J Clin Neurol 5: 95-96, 2009

8. Kitano M, Taneda M: Extended transsphenoidal approach with submucosal posterior ethmoidectomy for parasellar tumors. Technical note. J Neurosurg 94: 999-1004, 2001

9. McClain CD, Soriano SG, Goumnerova LC, Black PM, Rockoff MA: Detection of unanticipated intracranial hemorrhage during intraoperative magnetic resonance image-guided neurosurgery. Report of two cases. J Neurosurg 106: 398-400, 2007

10. Menon G, Bahuleyan B, Nair S: Acute subdural hematoma after transsphenoidal surgery. J Clin Neurosci 16: 160-162, 2009

11. Sciubba DM, Kretzer RM, Wang PP: Acute intracranial subdural hematoma following a lumbar CSF leak caused by spine surgery. Spine (Phila Pa 1976) 15: 730-732, 2005

12. Sharma S, Halliwell R, Dexter M, Mudaliar Y, Yee K: Acute subdural haematoma in the presence of an intrathecal catheter placed for the prevention of post-dural puncture headache. Anaesth Intensive Care 38: 939-941, 2010

13. Sudhakar N, Vafidis JA: Subdural haematoma after transsphenoidal surgery. Br J Neurosurg 17: 253-255, 2003

14. Tanaka Y, Kobayashi S, Hongo K, Tada T, Kakizawa Y: Chronic subdural hematoma after transsphenoidal surgery. J Clin Neurosci 9: 323-325, 2002

15. Yamada S, Fukuhara N, Oyama K, Takeshita A, Takeuchi $\mathrm{Y}$, Ito J, Inoshita N: Surgical outcome in 90 patients with craniopharyngioma: An evaluation of transsphenoidal surgery. World Neurosurg 74: 320-330, 2010 